\title{
Exact analytical solutions of the fractional biological population model, fractional EW and modified EW equations
}

\author{
Meryem Odabasi ${ }^{a, b, *}$ \\ ${ }^{a}$ Tire Kutsan Vocational School, Ege University, Turkey \\ ${ }^{b}$ Bioengineering Department, Faculty of Engineering, Ege University, Turkey \\ meryem.odabasi@ege.edu.tr, meryemodabasi@gmail.com
}

\section{ARTICLE INFO}

Article history:

Received: 8 July 2019

Accepted: 12 April 2020

Available Online: 17 December 2020

Keywords:

Fractional differential equations

Conformable derivative

Trial solution algorithm

Exact traveling wave solutions

AMS Classification 2010:

$34 \mathrm{A08}, 35 \mathrm{R} 11,35 \mathrm{CO}$

\begin{abstract}
In this paper, exact analytical solutions of the biological population model, the EW and the modified EW equations with a conformable derivative operator have been examined by means of the trial solution algorithm and the complete discrimination system. Dark, bright and singular traveling wave solutions of the equations have been obtained by algorithm. Also, revealed singular periodic solutions have been listed. All solutions were verified by substituting them into their corresponding equation via Mathematica package program.
\end{abstract}

\section{Introduction}

Due to the applications in nonlinear optics, biology, population dynamics, biomathematics, and other areas, fractional order differential equations have been considered more often. Differential equations of noninteger order construct more accurate models for the phenomena they describe. Hence, to find analytical and numerical solutions of these equations, some effective methods have been introduced and applied so far. Even so, a general method could not be proposed so scientists are still trying to develop new approaches. Finding exact analytical solutions of noninteger order differential equations play an essential role in describing the behavior of the considered model. This study implements the trial solution algorithm with the aid of complete discrimination system to establish exact solutions of non-integer order differential equations.

To this purpose, we first consider time fractional biological population model [1-4]. The equation describes population dynamics and gives ideas about complex interactions. Then, space-time fractional equal width (EW) equation [5] which describes complex physical phenomena in many fields has been considered. Finally, space-time fractional modified equal width equation [6] that describes the wave propagation with dispersion processes for one- dimensional nonlinear form was considered. Exact analytical solutions of the considered equations have been obtained successfully. All of these solutions have been confirmed by substituting them into their corresponding equation with the aid of Mathematica. Also, some solutions have been plotted with Maple to depict the structure of the solution equations. These models are quite important for mathematical physics and finding exact analtyical solutions of them may help the further analytical studies.

\section{Conformable fractional derivative and trial solution algorithm}

Fractional derivative reveals more suitable models for real world problems than integer order derivative. For this reason, many researchers have paid attention to develop new definitions of fractional derivative such as Caputo-Fabrizio [7] and Riemann-Liouville [8]. Also, a new definition, conformable derivative is proposed to overcome some setbacks of the existing derivatives, see Khalil et al. [9].

Conformable fractional derivative for a function $f$ of order $\alpha$ is defined as

$$
T_{\alpha}(f)(t)=\lim _{\tau \rightarrow 0} \frac{f\left(t+\tau t^{1-\alpha}\right)-f(t)}{\tau},
$$


where $f:[0, \infty) \rightarrow R$ for all $t>0$ and $\alpha \in(0,1)$ [9].

Some properties of the conformable fractional derivative are summarized as $[9,10]$ :

$T_{\alpha}(c f+k g)=c T_{\alpha}(f)+k T_{\alpha}(g)$, for all $c, k \in R$.

$T_{\alpha}\left(t^{\beta}\right)=\beta t^{\beta-\alpha}$, for all $\beta \in R$.

$T_{\alpha}(f \circ g)=t^{1-\alpha} f^{\prime}(g(t)) g^{\prime}(t)$.

Also, fractional versions of the Laplace transform, Taylor power series expansions and integration by parts are given by Abdeljawad, see [10].

Some authors [11-16] have studied fractional differential equations by different methods. Besides these, to investigate analytical solutions of PDEs, Liu proposed an approach called trial equation method which aims to reduce the examined equation to the solvable differential equations. Also Liu proposed complete discrimination system (CDS) to find exact solutions of PDEs, see [17-19]. The method is studied by some authors [20-23] to investigate analytical solutions of integer and non-integer order partial differential equations.

Solution steps of the trial solution algorithm can be outlined as follows [17-19]:

Step 1. We can consider a PDE of $u$. Using a wave transformation, it can be reduced to an ODE.

Step 2. Trial equation can be chosen as

$$
U^{\prime}=\sum_{i=1}^{n} a_{i} U^{i}
$$

or

$$
\left(U^{\prime}\right)^{2}=\sum_{i=1}^{n} a_{i} U^{i}
$$

according to the structure of the reduced equation. Using the balance procedure, the value of $n$ can be determined.

Step 3. Rewriting these equations into integral form

$$
\begin{aligned}
\pm\left(\xi-\xi_{0}\right) & =\int \frac{d U}{\sum_{i=1}^{n} a_{i} U^{i}} \\
\text { or } \quad \pm\left(\xi-\xi_{0}\right) & =\int \frac{d U}{\sqrt{\sum_{i=1}^{n} a_{i} u^{i}}},
\end{aligned}
$$

and using the CDS for polynomial yield the exact solution of the considered PDE.

We will apply this procedure for the fractional models.

\section{Governing models}

\subsection{The time fractional biological population model}

Consider the time fractional biological population model:

$$
\frac{\partial^{\alpha} u}{\partial t^{\alpha}}=\frac{\partial^{2}}{\partial x^{2}}\left(u^{2}\right)+\frac{\partial^{2}}{\partial y^{2}}\left(u^{2}\right)+h\left(u^{2}-r\right)
$$

where $t>0,0<\alpha \leq 1, x, y \in R$, and $h, r$ are constants. $u$ is the population density and $h\left(u^{2}-r\right)$ denotes the population supply as a result of births and deaths. [14].

Under the transformation

$$
u(x, y, t)=U(\xi), \quad \xi=k x+i k y-\frac{c t^{\alpha}}{\alpha},
$$

where $c, k$ are constants and $i^{2}=-1$, Eq.(6) turns into the following ODE:

$$
c U^{\prime}+h U^{2}-h r=0
$$

Balancing $U^{\prime}$ and $U^{2}$ yields $n=2$. Then trial equation is:

$$
U^{\prime}=a_{0}+a_{1} U+a_{2} U^{2} .
$$

Substituting Eq.(9) into Eq.(8) and letting all the coefficients of obtained polynomial to zero yields an algebraic equation system:

$$
\begin{aligned}
-h r+c a_{0} & =0 \\
c a_{1} & =0 \\
h+c a_{2} & =0 .
\end{aligned}
$$

From the system above, coefficients are determined as:

$$
a_{0}=\frac{h r}{c}, a_{1}=0, a_{2}=-\frac{h}{c} .
$$

Substituting these coefficients into Eq.(4) and integrating the equation gives the following exact traveling wave solutions of the Eq.(6):

$$
\begin{aligned}
& u(x, y, t)=\sqrt{r} \tanh \left[\frac{h}{c} \sqrt{r}\left(k x+i k y-c \frac{t^{\alpha}}{\alpha}\right)\right], \\
& u(x, y, t)=\sqrt{r} \operatorname{coth}\left[\frac{h}{c} \sqrt{r}\left(k x+i k y-c \frac{t^{\alpha}}{\alpha}\right)\right],
\end{aligned}
$$

which are dark and singular soliton solutions for $r>0$. The solutions (11) and (12) signify singular periodic solutions

$$
\begin{aligned}
& u(x, y, t)=-\sqrt{-r} \tan \left[\frac{h}{c} \sqrt{-r}\left(k x+i k y-c \frac{t^{\alpha}}{\alpha}\right)\right], \\
& u(x, y, t)=\sqrt{-r} \cot \left[\frac{h}{c} \sqrt{-r}\left(k x+i k y-c \frac{t^{\alpha}}{\alpha}\right)\right],
\end{aligned}
$$

as long as $r<0$.

Figures 1-2 represent the graphs of the solution Eq.(11) for some arbitrary constants. 


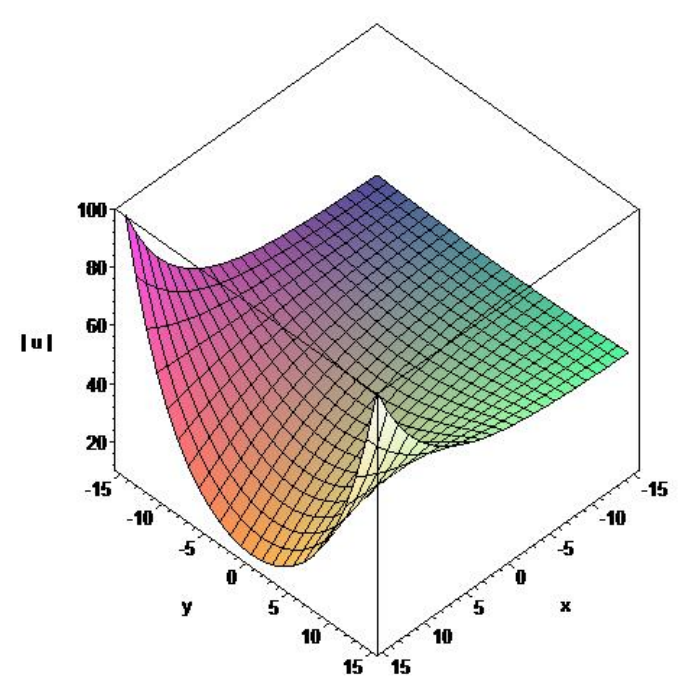

(a) Solution Eq.(11) for $\alpha=0.05$

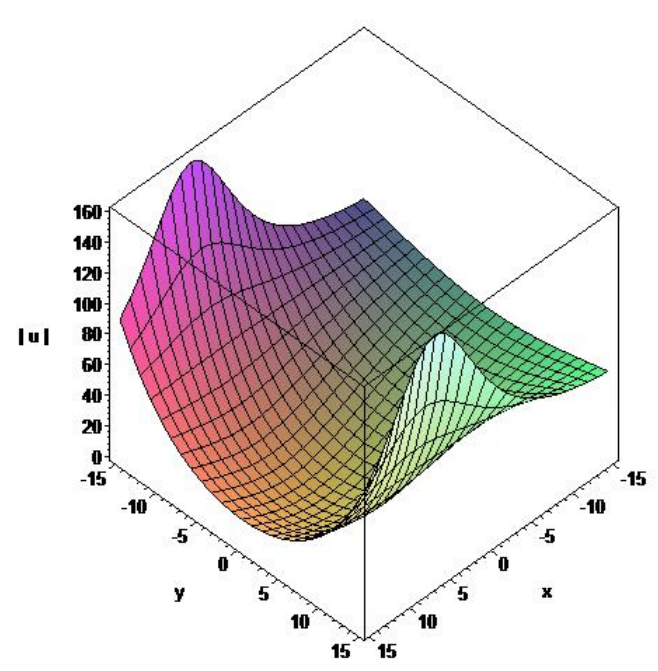

(b) Solution Eq.(11) for $\alpha=0.5$

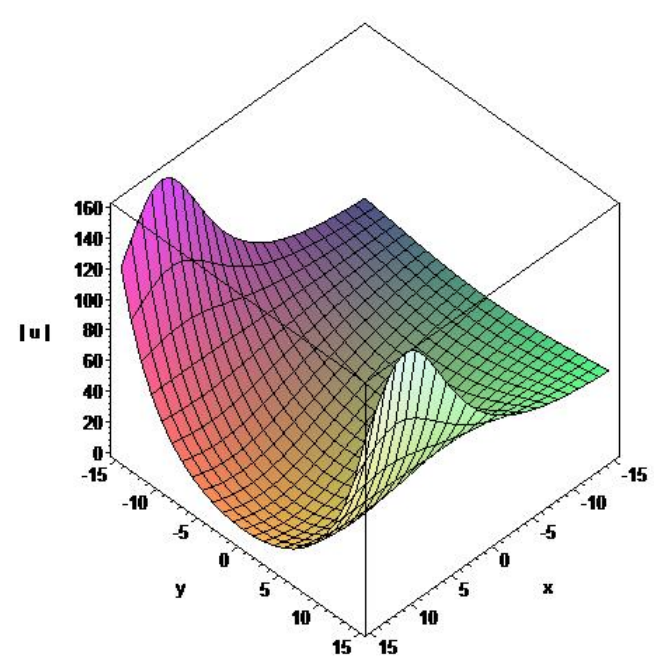

(c) Solution Eq.(11) for $\alpha=1$

Figure 1. Solution Eq.(11) for $h=0.01, r=50, c=k=1$, $t=10$ and indicated $\alpha$ values.

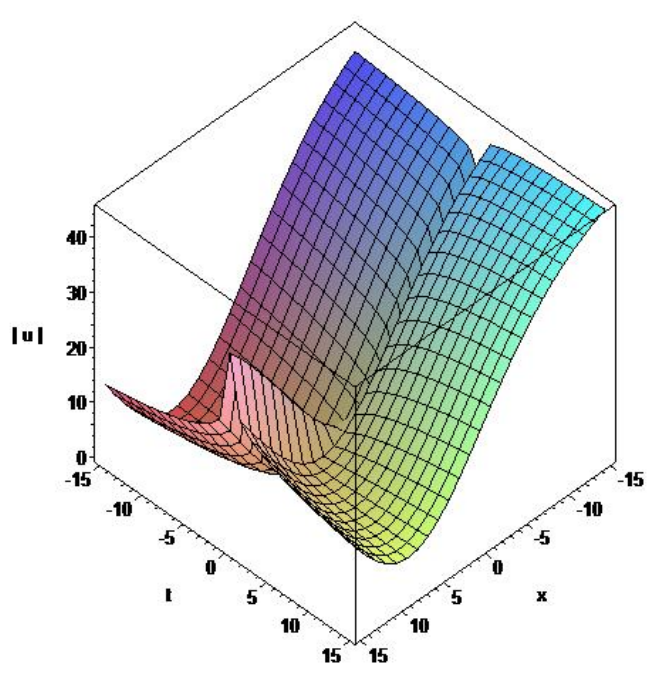

(a) Solution Eq.(11) for $\alpha=0.2$

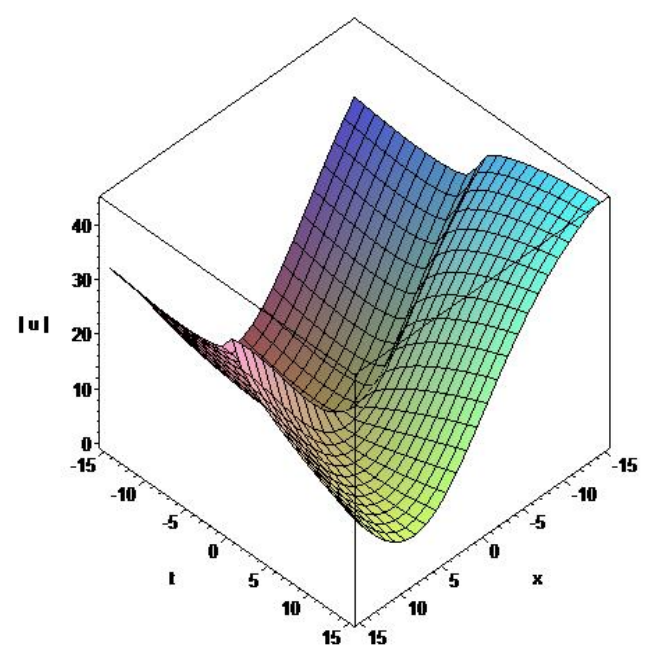

(b) Solution Eq.(11) for $\alpha=0.5$

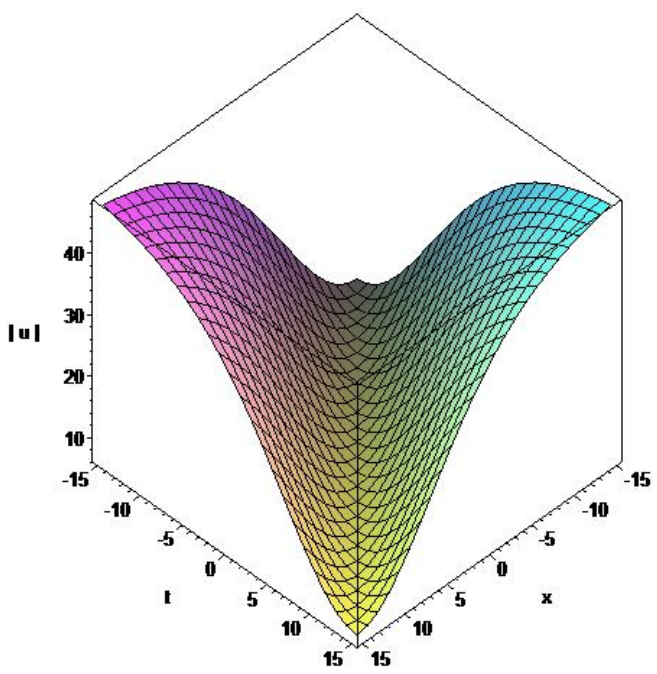

(c) Solution Eq.(11) for $\alpha=1$

Figure 2. Solution Eq.(11) for $h=0.01, r=50, c=k=1$, $y=5$ and indicated $\alpha$ values. 


\subsection{The space-time fractional equal width (EW) equation}

Consider the space-time fractional EW equation [5]

$$
D_{t}^{\alpha} u+\varepsilon D_{x}^{\alpha} u^{2}-\delta D_{x x t}^{3 \alpha} u=0
$$

where $t>0,0<\alpha \leq 1$, and $\varepsilon, \delta$ are real parameters. Under the transformation

$$
u(x, t)=U(\xi), \quad \xi=k \frac{x^{\alpha}}{\alpha}-c \frac{t^{\alpha}}{\alpha},
$$

Eq.(15) converts into the ODE:

$$
-c U^{\prime}+\varepsilon k\left(U^{2}\right)^{\prime}+\delta c k^{2} U^{\prime \prime \prime}=0
$$

Integrating Eq.(17)and letting the constant of integration zero yields

$$
-c U+\varepsilon k U^{2}+\delta c k^{2} U^{\prime \prime}=0 .
$$

Balancing $U^{\prime \prime}$ and $U^{2}$ results with $n=3$. Then, trial equation is:

$$
\left(U^{\prime}\right)^{2}=a_{0}+a_{1} U+a_{2} U^{2}+a_{3} U^{3} .
$$

With the same procedure, corresponding system of algebraic equation

$$
\begin{aligned}
c k^{2} \delta a_{1} & =0 \\
-2 c+c 2 k^{2} \delta a_{2} & =0 \\
2 k \varepsilon+3 c k^{2} \delta a_{3} & =0
\end{aligned}
$$

is obtained. So, the coefficients are determined as

$$
a_{0}=a_{0}, a_{1}=0, a_{2}=\frac{1}{\delta k^{2}}, a_{3}=-\frac{2 \varepsilon}{3 c k \delta} .
$$

Using the coefficients into Eq.(5) gives

$$
\pm\left(\xi-\xi_{0}\right)=\int \frac{d U}{\sqrt{a_{0}+\frac{1}{\delta k^{2}} U^{2}-\frac{2 \varepsilon}{3 c k \delta} U^{3}}} .
$$

Integrating (21) aid of the CDS, exact solutions of fractional $\mathrm{EW}$ equations have been emerged as traveling wave solutions along with $\delta>0$

$$
\begin{gathered}
u(x, t)=-\frac{3 c}{2 k \varepsilon} \operatorname{sech}^{2}\left[\frac{1}{2 k \sqrt{\delta}}\left(k \frac{x^{\alpha}}{\alpha}-c \frac{t^{\alpha}}{\alpha}\right)\right], \\
u(x, t)=\frac{3 c}{2 k \varepsilon} \operatorname{csch}^{2}\left[\frac{1}{2 k \sqrt{\delta}}\left(k \frac{x^{\alpha}}{\alpha}-c \frac{t^{\alpha}}{\alpha}\right)\right] .
\end{gathered}
$$

Solutions (22) and (23) mean periodic function solutions along with $\delta<0$

$$
\begin{aligned}
& u(x, t)=-\frac{3 c}{2 k \varepsilon} \sec ^{2}\left[\frac{1}{2 k \sqrt{-\delta}}\left(k \frac{x^{\alpha}}{\alpha}-c \frac{t^{\alpha}}{\alpha}\right)\right], \\
& u(x, t)=-\frac{3 c}{2 k \varepsilon} \csc ^{2}\left[\frac{1}{2 k \sqrt{-\delta}}\left(k \frac{x^{\alpha}}{\alpha}-c \frac{t^{\alpha}}{\alpha}\right)\right] .
\end{aligned}
$$

Figure 3 shows the graphs of the solution Eq.(22) for some values of the arbitrary constants.

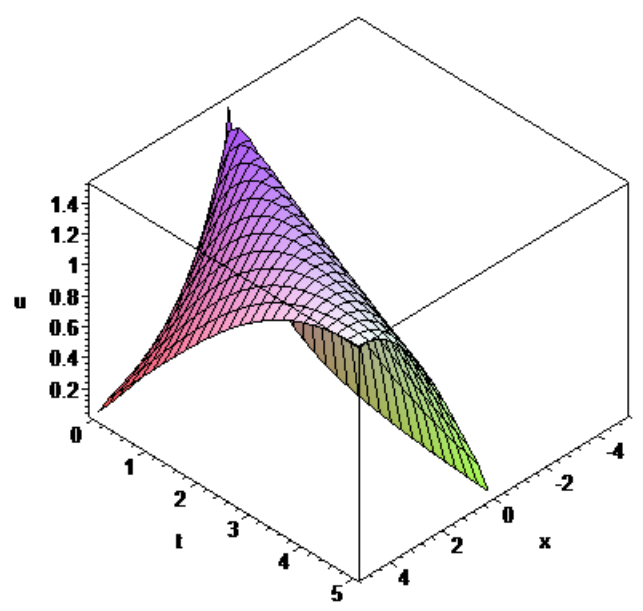

(a) Solution Eq.(22) for $\alpha=0.4$

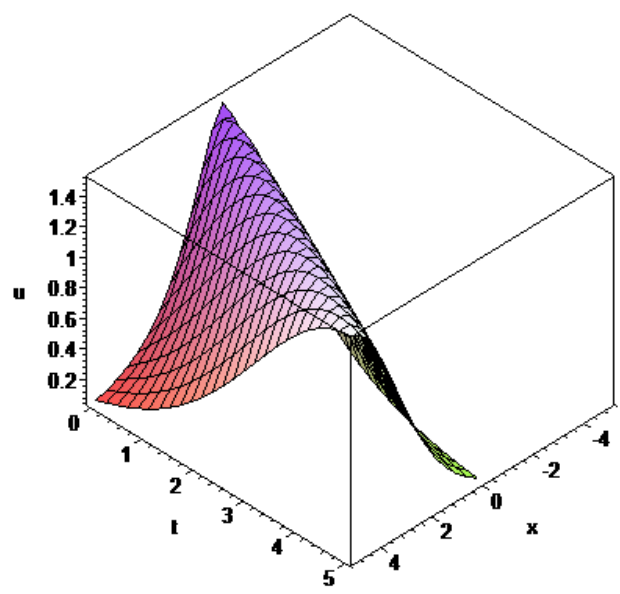

(b) Solution Eq.(22) for $\alpha=0.8$

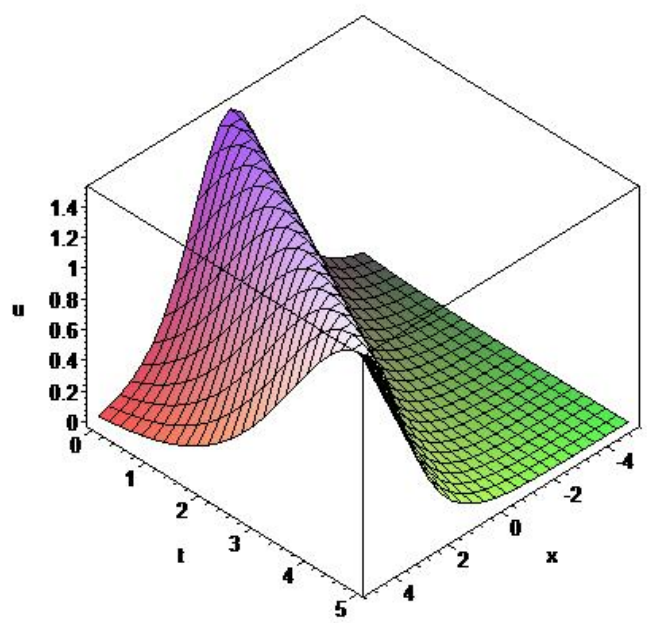

(c) Solution Eq.(22) for $\alpha=1$

Figure 3. Solution Eq.(22) for $c=k=\delta=1, \varepsilon=-1$ and indicated $\alpha$ values. 


\subsection{The space-time fractional modified EW} equation

Now we consider the space-time fractional modified EW equation [6]

$$
D_{t}^{\alpha} u+\varepsilon D_{x}^{\alpha} u^{3}-\delta D_{x x t}^{3 \alpha} u=0,
$$

where $t>0$ and $0<\alpha \leq 1$.

Using the transformation (16), Eq.(26) reduces into the ODE:

$$
-c U^{\prime}+\varepsilon k\left(U^{3}\right)^{\prime}+\delta c k^{2} U^{\prime \prime \prime}=0 .
$$

Integrating Eq.(27) and letting the constant of integration to zero gives:

$$
-c U+\varepsilon k U^{3}+\delta c k^{2} U^{\prime \prime}=0 .
$$

Balancing the terms $U^{\prime \prime}$ and $U^{3}$ gives $n=4$. Trial equation can be chosen as

$$
\left(U^{\prime}\right)^{2}=a_{0}+a_{1} U+a_{2} U^{2}+a_{3} U^{3}+a_{4} U^{4} .
$$

If one uses the same procedure above, corresponding system

$$
\begin{aligned}
c k^{2} \delta a_{1} & =0 \\
-2 c+c 2 k^{2} \delta a_{2} & =0 \\
3 c k^{2} \delta a_{3} & =0 \\
2 k \varepsilon+4 c k^{2} \delta a_{4} & =0
\end{aligned}
$$

is obtained. So, the coefficients are determined as

$$
a_{0}=a_{0}, a_{1}=0, a_{2}=\frac{1}{\delta k^{2}}, a_{3}=0, a_{4}=-\frac{\varepsilon}{2 c k \delta} \text {. }
$$

Using the coefficients into Eq.(5) and applying solution procedure of the trial solution algorithm, exact solutions to the modified EW equation are determined as bright and dark traveling wave solutions for when $\delta>0$

$$
\begin{gathered}
u(x, t)=\sqrt{\frac{-2 c}{\varepsilon k}} \operatorname{sech}\left[\frac{1}{k \sqrt{\delta}}\left(k \frac{x^{\alpha}}{\alpha}-c \frac{t^{\alpha}}{\alpha}\right)\right], \\
u(x, t)=\sqrt{\frac{2 c}{\varepsilon k}} \operatorname{csch}\left[\frac{1}{k \sqrt{\delta}}\left(k \frac{x^{\alpha}}{\alpha}-c \frac{t^{\alpha}}{\alpha}\right)\right] .
\end{gathered}
$$

Solutions (31) and (32) purport singular periodic function solutions provided that $\delta<0$

$$
\begin{aligned}
& u(x, t)=\sqrt{\frac{-2 c}{\varepsilon k}} \sec \left[\frac{1}{k \sqrt{-\delta}}\left(k \frac{x^{\alpha}}{\alpha}-c \frac{t^{\alpha}}{\alpha}\right)\right], \\
& u(x, t)=\sqrt{\frac{-2 c}{\varepsilon k}} \csc \left[\frac{1}{k \sqrt{-\delta}}\left(k \frac{x^{\alpha}}{\alpha}-c \frac{t^{\alpha}}{\alpha}\right)\right] .
\end{aligned}
$$

Figures 4-5 represent graphs of the solutions (31) and (33).

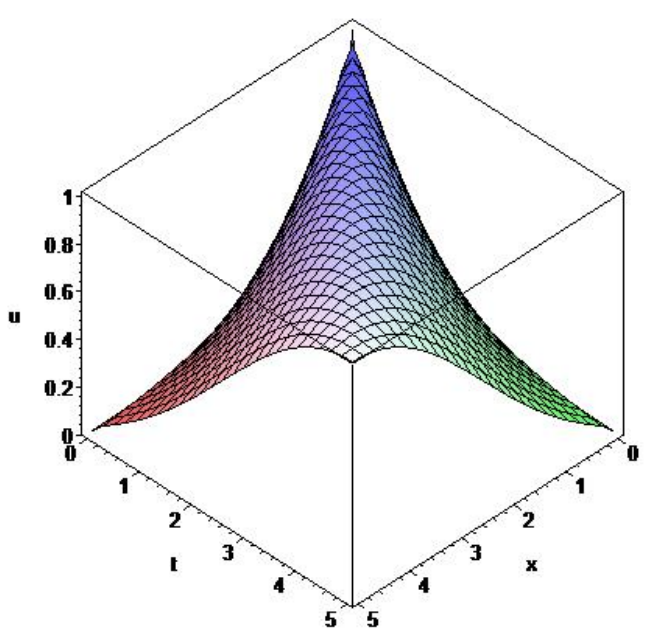

(a) Solution Eq.(31) for $\alpha=0.4$

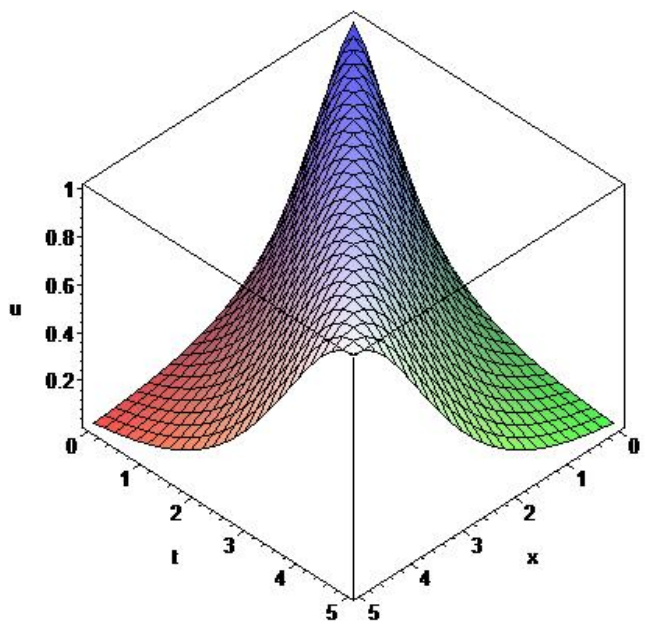

(b) Solution Eq.(31) for $\alpha=0.8$

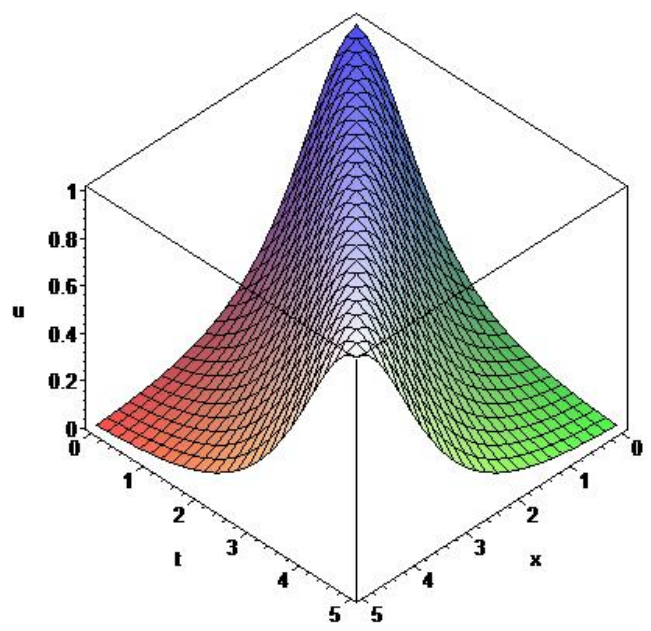

(c) Solution Eq.(31) for $\alpha=1$

Figure 4. Solution Eq.(31) for $c=k=\delta=1, \varepsilon=-2$ and indicated $\alpha$ values. 


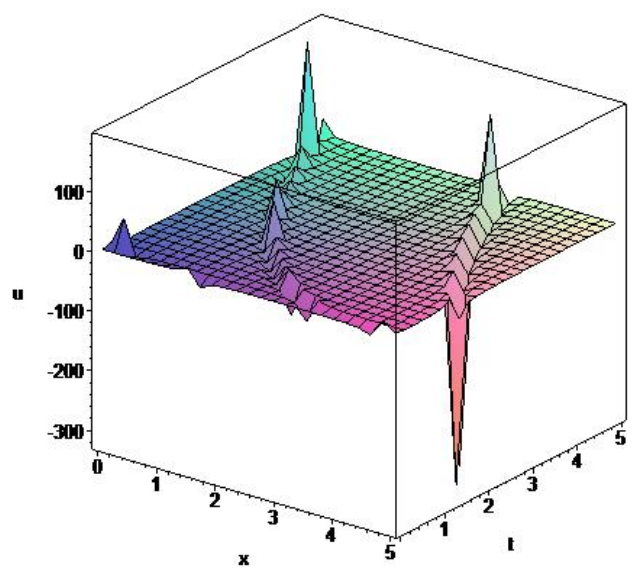

(a) Solution Eq.(33) for $\alpha=0.4$

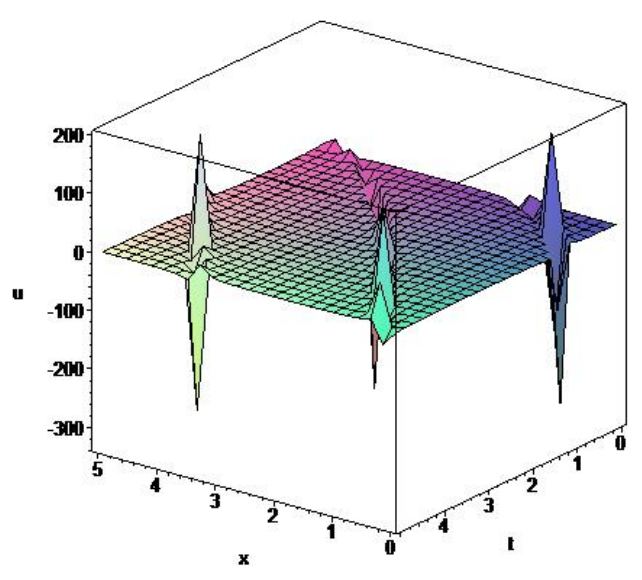

(b) Solution Eq.(33) for $\alpha=0.8$

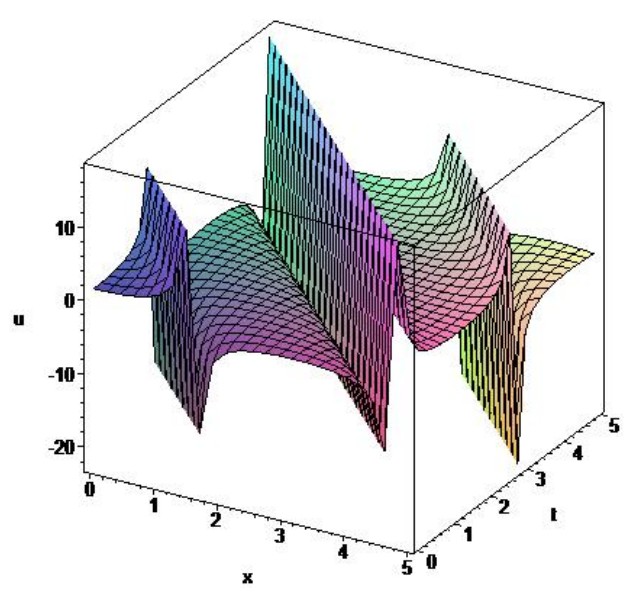

(c) Solution Eq.(33) for $\alpha=1$

Figure 5. Solution Eq.(33) for $k=\varepsilon=1, c=\delta=-1$ and indicated $\alpha$ values.

\section{Conclusion}

The trial solution algorithm with aid of CDS was implemented to find exact traveling wave solutions of the fractional biological population model, the fractional EW and the fractional modified EW equations in sense of conformable derivative. Using this algorithm, significant dark, bright and also singular periodic traveling wave solutions of these equations were obtained. All solutions were checked by Mathematica and some of them were plotted by Maple. Finding exact analytical solutions of the models may play a quite important role for explaining the physical phenomenon they characterize.

\section{Acknowledgments}

The author would like to thank to Ege University, Scientific Research Project (BAP) for the support on the Project 17-TKMYO-002.

\section{References}

[1] El-Sayed, A.M.A., Rida, S.Z., Arafa, A.A.M. (2009). Exact solutions of fractional-order biological population model. Communications in Theoretical Physics, 52, 992-996.

[2] Bekir, A., Güner, Ö., Cevikel, A.C. (2013). Fractional complex transform and exp-function methods for fractional differential equations. Abstract and Applied Analysis, Article ID 426462, 8 pages, DOI: $10.1155 / 2013 / 426462$.

[3] Kumar, D., Seadawy, A.R., Joardar, A.K. (2018). Modified Kudryashov method via new exact solutions for some conformable fractional differential equations arising in mathematical biology. Chinese Journal of Physics, 56, 75-85.

[4] Meng, F. (2013). A New Approach for solving fractional partial differential equations. Journal of Applied Mathematics, Article ID 256823, 5 pages, DOI: $10.1155 / 2013 / 256823$.

[5] Hosseini, K., Ayati, Z. (2016). Exact solutions of space-time fractional EW and modified EW equations using Kudryashov method. Nonlinear Science Letters A,7 (2), 58-66.

[6] Guner, O., Bekir, A. (2017). A novel method for nonlinear fractional differential equations using symbolic computation. Waves in Random and Complex Media, 27(1), 163-170.

[7] Caputo, M., Fabrizio, M. (2015). A new definition of fractional derivative without singular kernel. Progress in Fractional Differentiation and Applications, 1(2), 73-85.

[8] Shadab, M., Faisal Khan, M., Luis Lopez-Bonilla, J. (2018). A new Riemann-Liouville type fractional derivative operator and its application in generating functions. Advances in Difference Equations, 167.

[9] Khalil, R., Horani, M.A., Sababheh A.M.Y. (2014). A new definition of fractional derivative. Journal of Computational and Applied Mathematics ,264, 65- 
70

[10] Abdeljawad, T. (2015). On conformable fractional calculus. Journal of Computational and Applied Mathematics, 279, 57-66.

[11] Hosseini, K., Korkmaz, A., Bekir, A., Samadani, F., Zabihi, A., Topsakal, M. (2019). New wave form solutions of nonlinear conformable timefractional Zoomeron equation in $(2+1)$ dimensions. Waves in Random and Complex Media, DOI: 10.1080/17455030.2019.1579393.

[12] Hosseini, K., Manafian, J., Samadani, F., Foroutan, M., Mirzazadeh, M., Zhou, Q. (2018). Resonant optical solitons with perturbation terms and fractional temporal evolution using improved tan $(\phi(\eta) / 2)$-expansion method and exp function approach. Optik, 158, 933-939.

[13] Hosseini, K., Mayeli, P., Bekir, A., Guner, O. (2018). Density-dependent conformable space-time fractional diffusion-reaction equation and its exact solutions. Communications in Theoretical Physics, $69,1-4$.

[14] Odabasi, M., Pinar, Z., Kocak, H. (2020). Analytical solutions of some nonlinear fractionalorder differential equations by different methods. Mathematical Methods in the Applied Sciences, DOI: $10.1002 / \mathrm{mma} .6313$.

[15] Hosseini, K., Bekir, A., Kaplan, M., Guner, O. (2017). On a new technique for solving the nonlinear conformable time-fractional differential equations. Optical and Quantum Electronics, 49, 343.

[16] Hosseini, K., Bekir, A., Ansari, R. (2017). Exact solutions of nonlinear conformable time-fractional Boussinesq equations using the $\exp (-\phi(\varepsilon))$ expansion method. Optical and Quantum Electronics, 49, 131.

[17] Liu, C.S. (2006). Trial equation method to nonlinear evolution equations with rank inhomogeneous: mathematical discussions and its applications. Communications in Theoretical Physics, 45, 219-223.

[18] Liu, C.S. (2006). A New trial equation method and its applications. Communications in Theoretical Physics, 45, 395-397.

[19] Liu, C.S. (2010). Applications of complete discrimination system for polynomial for classifications of traveling wave solutions to nonlinear differential equations. Computer Physics Communications, 181, 317-324.

[20] Biswas, A. (2018). Optical solitons with differential group delay for coupled Fokas-Lenells equation by extended trial function scheme. Optik, 165, 102110 .

[21] Odabasi, M., Misirli, E. (2017). A note on the traveling wave solutions of some nonlinear evolution equations. Optik, 142, 394-400.

[22] Odabasi, M., Misirli, E. (2018). On the solutions of the nonlinear fractional differential equations via the modifed trial equation method. Mathematical Methods in the Applied Sciences, 41, 904-911.

[23] Odabasi, M. (2020). Traveling wave solutions of conformable time-fractional Zakharov-Kuznetsov and Zoomeron equations. Chinese Journal of Physics, 64, 194-202.

Meryem Odabasi received her M.Sc. and Ph.D. degrees from Applied Mathematics, Ege University in 2009 and 2015, respectively. She currently works at Ege University as a lecturer. Her research areas are analytical and numerical methods for nonlinear differential equations. iD http://orcid.org/0000-0002-3025-3063

An International Journal of Optimization and Control: Theories \& Applications (http://ijocta.balikesir.edu.tr)

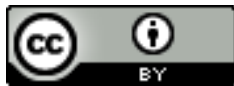

This work is licensed under a Creative Commons Attribution 4.0 International License. The authors retain ownership of the copyright for their article, but they allow anyone to download, reuse, reprint, modify, distribute, and/or copy articles in IJOCTA, so long as the original authors and source are credited. To see the complete license contents, please visit http://creativecommons.org/licenses/by/4.0/. 